\title{
"humanidades
}

Revista Humanidades

ISSN: 2215-3934

humanidades@ucr.ac.cr

Universidad de Costa Rica

Costa Rica

\section{Estrategias eficaces de práctica instrumental. Primeros pasos al estudiar una obra musical de Silvia Tripiana Muñoz, Editorial Libargo}

Gisbert Caudeli, Dra. Vicenta

Estrategias eficaces de práctica instrumental. Primeros pasos al estudiar una obra musical de Silvia Tripiana Muñoz, Editorial Libargo

Revista Humanidades, vol. 12, núm. 1, e48864, 2022

Universidad de Costa Rica, Costa Rica

Disponible en: https://www.redalyc.org/articulo.oa?id=498068490006 DOl: https://doi.org/10.15517/h.v12i1.48864

\section{(c) (1) @ $\Theta$}

Esta obra está bajo una Licencia Creative Commons Atribución-NoComercial-SinDerivar 3.0 Internacional. 


\title{
Estrategias eficaces de práctica instrumental. Primeros pasos al estudiar una obra musical de Silvia Tripiana Muñoz, Editorial Libargo
}

\author{
Effective Instrumental Practice Strategies. First Steps when Studying a Musical Work by Silvia Tripiana Muñoz, \\ Libargo Publisher
}

Dra. Vicenta Gisbert Caudeli

Universidad Internacional de La Rioja, Logroño, España

vicenta.gisbert@unir.net

(iD) https://orcid.org/0000-0003-1763-1143

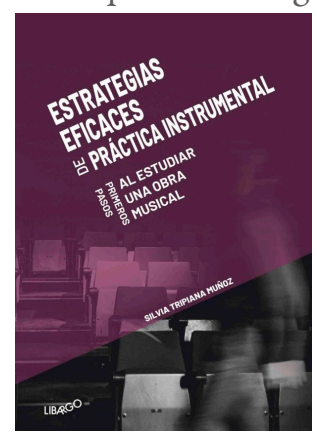

DOI: https://doi.org/10.15517/h.v12i1.48864

Redalyc: https://www.redalyc.org/articulo.oa? id $=498068490006$ Tripiana Silvia. Estrategias eficaces de práctica instrumental. Primeros
pasos al estudiar una obra musical. 2019. Granada. Editorial Libargo.
184pp.. 97884948136658494813668

Siguiendo con la metáfora farmacéutica, desgranaremos los ingredientes fundamentales de esta fórmula educativa de la mano de esta licenciada en Medicina y Cirugía que, además de preocuparse por la sanación física, se ha preocupado por incorporar los beneficios espirituales que, como bien decía Platón, nos proporcionan la armonía musical acercándonos a la virtud. En su formación como titulada Superior de Piano, Música de Cámara, Solfeo, Teoría de la Música, Transposición y Acompañamiento se sobreentiende una vocación por la enseñanza y un compromiso y preocupación por guiar adecuadamente al alumnado en sus avances musicales. En este ejemplar, encontramos implícito el interés por buscar las múltiples visiones posibles con las que abordar el estudio de una obra musical desde sus inicios. Desde la estrategia fragmentada hasta la estrategia analítica, pasando por la ergonomía, la auto-orientación o la práctica indirecta, entre otras. Todo ello, elucidado desde la vivencia en primera persona y el estudio reflexivo de los resultados obtenidos en su extensa labor docente.

En estas páginas dejamos a un lado el talento, del que tanto se habla en las manifestaciones artísticas (y que únicamente poseen los elegidos), proporcionándole protagonismo a la igualdad de oportunidades y garantizando el acceso al arte musical a todos aquellos que deseen embarcarse en tan noble propósito. La perseverancia y la pasión guiarán al futuro intérprete en el estudio musical, hasta hacer del hecho musical un medio de comunicación emocional con cuidada expresión artística y personal vivencia interpretativa. Se observa en este trabajo un amplio repertorio de recursos reales con una característica común: todos son factibles hacia una meta final, en este caso, la optimización del tiempo de estudio, así como la obtención del máximo nivel musical posible en cada individuo.

A pesar de los beneficios constatados del aprendizaje por imitación (higiene postural, actitud escénica, precisión técnica y correcta afinación, estilo interpretativo, etc.), en este libro se aboga por otras estrategias a implementar en la docencia. La primera de ellas es la fragmentación, la cual está precedida del análisis selectivo de dificultades. Esta técnica consiste en desgranar la dificultad para emprender su estudio de forma aislada e integrarlo conectivamente en el discurso musical de forma progresiva. No se refiere únicamente 
a la división de pasajes musicales, sino a una secuenciación de tempi (graduando la dificultad conforme se incrementa la velocidad de ejecución) que implique un reto interpretativo. Ha de resultar un recurso al alcance del alumno, porque si fuera inalcanzable provocaría frustración y deseos de abandonar la obra. Sería posible plantear el estudio de la memorización y la coordinación dactilar para la incorporación posterior de la exigencia tonal. La descomposición de los distintos parámetros musicales (ritmo, articulación, matices...) y su estudio interconectado favorecen la superación de las dificultades, lo que evita la obsesión y la focalización en el centro del problema.

Ahora bien, la práctica con máxima concentración es la segunda estrategia presentada. Dicho ejercicio alcanza su momento álgido cuando el desarrollo musical resulta absorbente. Esto genera una sensación de control y disfrute. Si se hubiera de encontrar un punto convergente en las técnicas interpretativas de los grandes solistas sería, sin lugar a duda, la concentración. El hecho de pretender alcanzar un elevado grado de concentración suele evitar la propia concentración. La autora aconseja intercalar pausas entre el estudio, así como abordarlo en un estado sereno físico y mental, buscando la optimización y calidad interpretativa sin obsesionarse por la cantidad de tiempo invertido.

La tercera estrategia es la ergonómica, la cual se fundamenta en el aprovechamiento del cuerpo con naturalidad y comodidad para incrementar, mediante el equilibrio y la armonía del método Willems y la relajación del método Martenot, la eficacia del estudio. Este recurso es un magnífico aliado en la prevención de la tensión corporal, que suele repercutir en la inconveniente tensión interpretativa. El control respiratorio, la higiene postural, un buen apoyo y la ayuda del silencio interior son algunos de los ingredientes imprescindibles en este método.

Los mensajes de auto-orientación resultan potentes elementos de mejora del rendimiento, pues contribuyen con la fijación de los objetivos mediante mensajes recordatorios. Estos últimos funcionan como retroalimentación evaluativa de la sesión de estudio. Los mensajes elaborados en cada intervalo de práctica musical, determinan la planificación de las sesiones posteriores en las que se integran e interiorizan rápidamente los cambios observados. La escucha autocrítica es concebida como un método de búsqueda de la excelencia interpretativa que potencia la motivación y el desarrollo musical. El estudiante de música posee instintivamente un desarrollado espíritu crítico, pero la percepción durante la interpretación no permite la objetividad. Las grabaciones y su posterior escucha y autocrítica permiten una valoración fiable del resultado musical. De esta manera, no es lo mismo interpretar música que estudiar la interpretación de nuestra música.

Como cualquier idioma, la música está condicionada al conocimiento, comprensión e interpretación de su código. La autora presenta su sexta estrategia, centrada en la precisión de la lectura musical. En este sentido, invita a reflexionar al lector sobre los errores cometidos en una interpretación. Aunque parece que una única nota equivocada podría repercutir negativamente en la valoración del intérprete más que todas las notas acertadas, esta situación es una realidad a la que todos los que han estudiado música se enfrentan. Ante el error, Tripiana plantea tres respuestas: en primer lugar, evitar centrarse en el error y proseguir la interpretación; en segundo lugar, detenerse al detectar el error para corregirlo y estudiar por secciones evitando cometer errores y; en tercer lugar, repetir la sección completa en caso de que se produzcan errores. El dominio de los pasajes de mayor dificultad conlleva precisión en la lectura del fragmento. De esta manera, se evitan errores ante el posible incremento ansiógeno en el escenario. Los efectos de la fijación de hábitos (mielinización), como apunta la autora, pueden resultar positivos si se inicia el estudio mediante la práctica lenta, promoviendo los buenos hábitos desde el primer contacto con la obra. La corrección de malas costumbres requiere de un esfuerzo innecesario. Además, esto podría ser causa de abandono del estudio, producto de la frustración y la desmotivación.

La penúltima estrategia es la práctica indirecta, la cual consiste en alterar la interpretación. Esto proporciona nuevas y mayores dificultades; para ello se puede variar el tempo de menor a mayor rapidez, realizar combinaciones rítmicas, alterar la acentuación, etc. El incremento de la dificultad para la interpretación posterior equivaldría a entrenar con peso en las extremidades superiores para competir sin 
ellas. De este modo, al superar la interpretación con dificultad incrementada, se obtiene solvencia en la interpretación original y se refuerza la seguridad al abordar los pasajes que implican mayor complicación.

Esta exposición de estrategias concluye con la denominada como respeto al límite actual. En esta estrategia se deben abandonar las ambiciones y un perfeccionamiento excesivo (relacionado con la ansiedad escénica), respetar el ritmo propio de evolución en la interpretación y evitar la elección de repertorio con exigencias por encima de las posibilidades individuales. De igual manera, se prestará especial atención a las tensiones musculares generadas, síntomas de sobreesfuerzo. Esto para encontrar la práctica más natural y cómoda, así como para evadir las exigencias innecesarias (matices, velocidades o acentuaciones al límite de las posibilidades). También, se evitará magnificar los errores e imponerse autocastigos si no se logran los objetivos marcados. No se atenderán los pensamientos negativos simultáneos a la interpretación.

La doctora Tripiana satisface a los lectores, amantes de la síntesis, incorporando, al finalizar la presentación de cada estrategia, un extraordinario cuadro-resumen con unos apartados de gran utilidad: definición aclaratoria sobre cada estrategia, propuestas más interesantes desarrolladas en el capítulo, mapa conceptual con los aspectos más relevantes y resultados que se pretenden obtener con su aplicación. Atesoran estas páginas años de observación, investigación y compromiso, maravillosamente aderezados con más de ciento cincuenta citas procedentes de grandes intérpretes y pedagogos musicales de referencia.

Así, este compendio de estrategias de aprendizaje se convierte en libro de cabecera de docentes responsables. No se trata de implementar estos recursos como si de un tutorial se tratase, sino de aplicar con sentido común los recursos adecuados a cada intérprete, situación y circunstancia. Recae en manos de los docentes una gran responsabilidad: la formación musical de las futuras generaciones de profesionales. Afortunadamente, la dedicación de expertos, como Silvia Tripiana, permite seguir avanzando en el incremento cualitativo de esta disciplina artística imprescindible en la formación humana, permitiendo como dice la autora "fomentar la igualdad de oportunidades de cada niña o niño que se aventure a comenzar a tocar un instrumento y ponga todas sus ilusiones en él” (p. 13).

\section{ReFERENCIAS BIBLIOGRÁFICAS}

Tripiana, S. (2019). Estrategias eficaces de práctica instrumental. Primeros pasos al estudiar una obra musical. Editorial Libargo. 